\title{
Study on Generation of Harmonic Voltage using Synchronous Machine with d-axis and q-axis Harmonic Field Windings
}

\author{
Eiichi Mukai *, Toshio Kakinoki *, Hitoshi Yamaguchi *, \\ Yoshimasa Kimura *, and Sumio Fukai **
}

\begin{abstract}
We examined the generation of harmonic voltage by a synchronous machine adding d-axis and q-axis harmonic field windings in order to reduce the harmonics in a power line. We derived the expressions of the armature voltage in the case of supplying the currents with the frequency $\mathrm{nf}$ to the $\mathrm{d}$-axis and $\mathrm{q}$-axis harmonic field windings. We constructed the synchronous machine adding the harmonic windings. In this paper, the expressions and the experimental results on the generation of harmonic voltages by the synchronous machine are presented.
\end{abstract}

\section{Keywords: Harmonic voltage, Synchronous machine, Harmonic field winding, Symmetric} coordinate system

\section{Introduction}

With the popularization of equipment using power semiconductors, harmonics have become a significant problem in power systems. Harmonics in power systems cause undesirable effects in the electrical machinery and apparatus on the load side. In particular, it is very important to remove low order harmonics such as the 5th and 7th harmonics, because they have a large influence on the electrical machinery and apparatus.

An LC filter is generally used to reduce the harmonics, but the LC filter is subjected the influence of the impedance value, which changes with the load condition in the power system. The LC filter may also succumb to burnout by overload. Therefore, an active filter has been studied to avoid these disadvantages in the LC filter [1]. However, the active filter will generate other harmonics in a different order because of the switching of the semiconductor. Moreover, there is a problem of the costs involved in the enlargement.

An absorption method of the power line harmonics using a synchronous machine has recently been investigated [2]. A method for practical application, however, has not yet been realized. When the synchronous machine is used for

\footnotetext{
Dept. of Computer and Information Science, Sojo University, Japan. (mukai@ cis.sojo-u.ac.jp)

** Dept. of Electrical and Electronics Engineering, Saga University, Japan. (fukais@cc.saga-u.ac.jp)

Received 13 April 2013; Accepted 15 May 2013
}

reducing the harmonics, the equipment using the synchronous machine is expected to be more advantageous than the LC filter and the active filter in terms of the cost.

In this paper, the armature voltages are presented in the case of applying an $\mathrm{AC}$ voltage with angler frequency $n \omega$ to the d-axis harmonic field winding of a synchronous machine. Then, it is shown that harmonic voltages with angler frequency $(n-1) \omega$ and $(n+1) \omega$ are induced in the armature winding. Moreover, the equations of the harmonic voltage generated in the armature winding are shown in the case of supplying simultaneously the currents to the d-axis and q-axis harmonic field windings.

We constructed a synchronous machine to ultimately demonstrate the reduction of the harmonics in a power line [3]-[4]. The structure of this experimental synchronous machine is different from a usual synchronous machine and the experimental machine has both $\mathrm{d}$-axis and q-axis field windings.

The power spectrum of the armature voltage was measured in the case of supplying the AC currents with frequency $f$ to the harmonic field windings of the experimental synchronous machine. Then, it was shown that the ratios of the amplitudes of harmonic voltages with frequency $(n-1) f$ and $(n+1) f$ have the possibility to change.

\section{Model of Synchronous Machine}

Fig. 1 shows the winding diagram of a synchronous 
machine with dual harmonic field windings $\mathrm{d} 2$ and $\mathrm{q} 2$.

In this figure, $\mathrm{a}, \mathrm{b}$ and $\mathrm{c}$ are 3-hase armature windings to absorb the harmonics contained in the power line. The winding $\mathrm{d} 1$ on the $\mathrm{d}$-axis is the ordinary field winding.

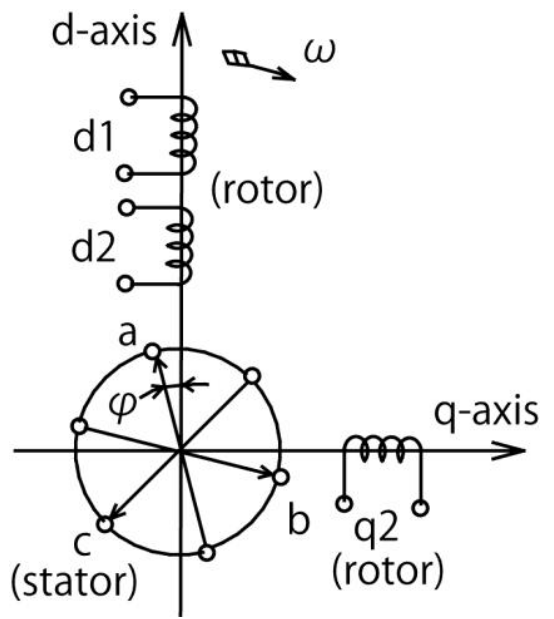

Fig. 1. Winding diagram of synchronous machine

In this figure, $a, b$ and $c$ are 3-phase armature windings to absorb the harmonics contained in the power line. The winding $\mathrm{d} 1$ on the $\mathrm{d}$-axis is the ordinary field winding. $\varphi$ is the electrical angle between the a-phase armature winding axis and the d-axis at $t=0$. The DC voltage is applied to the field winding $\mathrm{d} 1$. Moreover, AC voltages with the frequency $n f$ are applied to the harmonic field windings $\mathrm{d} 2$ and $\mathrm{q} 2$.

The design parameters of the experimental synchronous machine are given in Table 1.

Table 1. Design parameters of experimental synchronous machine

\begin{tabular}{|c|c|}
\hline Frequency & $60 \mathrm{~Hz}$ \\
\hline Voltage & $200 \mathrm{~V}$ \\
\hline Current & $1.9 \mathrm{~A}$ \\
\hline Power & $500 \mathrm{~W}$ \\
\hline Number of Poles & 4 \\
\hline Speed & $1800 \mathrm{rpm}$ \\
\hline
\end{tabular}

\section{Expression on Harmonic Voltage of Armature Winding}

In this section, the harmonic voltages of the armature winding are shown in the case of supplying AC currents to the harmonic windings $\mathrm{d} 2$ and $\mathrm{q} 2$.

We set the following assumptions:

(1) The windings of the synchronous machine under consideration are circumferentially distributed for the sinusoid.
(2) The rotor is rotating with the constant angular velocity $\omega$.

(3) The initial phase difference $\psi$ between the d-axis and the a-phase armature winding is zero for the simplicity.

The AC voltage $v_{d 2 n}$ with angular frequency $\omega$ is applied to the winding $\mathrm{d} 2$, and then the current $i_{d 2 n}$ as shown in (1) flows into the winding $\mathrm{d} 2$.

$$
i_{d 2 n}=I_{d 2 n} \cos \left(n \omega t+\phi_{n d}\right)
$$

where $I_{d 2 n}$ is the maximum value of $i_{d 2 n}$ and $\Psi_{\text {nd }}$ is the initial electrical angle at $t=0$.

This AC current $i_{d 2 n}$ can be resolved into two rotating currents $i_{d 2 F}$ (positive-phase-sequence component) and $i_{d 2 B}$ (negative-phase-sequence component) as (2), and each amplitude of the currents is one-half the maximum amplitude of $i_{d 2 n}$.

$$
i_{d 2 n}=i_{d 2 F}+i_{d 2 B}
$$

As shown in Fig. $2, i_{d 2 F}$ rotates with angular velocity $n \omega$ toward the revolution of the rotor and $i_{d 2 B}$ reversely rotates with $n \omega$.

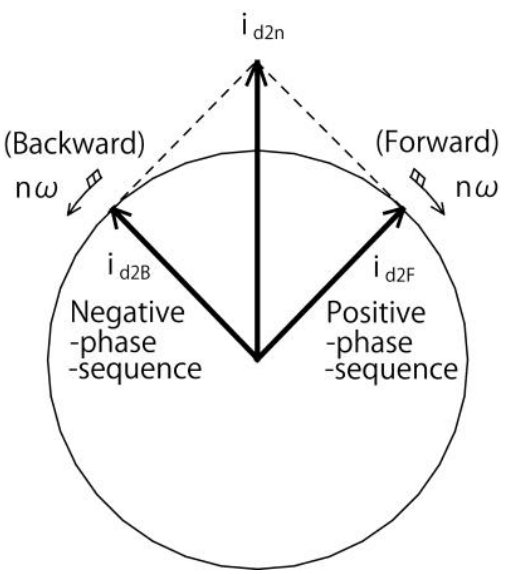

Fig. 2. Resolved currents into positive- and negativephase-sequence components

Since the magnetic flux due to $i_{d 2 F}$ rotates in the same direction as the rotor revolution, it is observed on the armature winding as magnetic flux rotating with $(n+1) \omega$ as shown in Fig. 3 (a). Therefore, voltage with angler frequency $(n+1) \omega$ is generated in the armature winding.

The harmonic voltage $v_{a d}(n+1)$ of the a-phase armature winding is expressed as the following equation.

$$
\begin{aligned}
v_{a d(n+1)}=(n+1) \omega & \cdot M_{a d 2} \cdot\left(I_{d 2 n} / 2\right) \\
& \times \cos \left\{(n+1) \omega t+\phi_{n d}\right\}
\end{aligned}
$$


where $\mathrm{M}_{\mathrm{ad} 2}$ is the mutual inductance between the a-phase armature winding and the winding $\mathrm{d} 2$.

In contrast, since the magnetic flux due to $i_{d 2 B}$ rotates in the inverse direction to the rotor revolution, it is observed on the armature winding as magnetic flux rotating with $(n-1) \omega$ as shown in Fig. 3 (b). Therefore, the harmonic voltage with angler frequency $(n-1) \omega$ is generated in the armature winding.

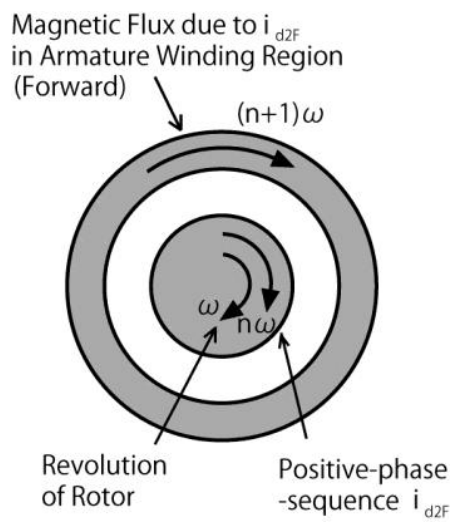

(a) Magnetic flux due to $i_{d 2 F}$

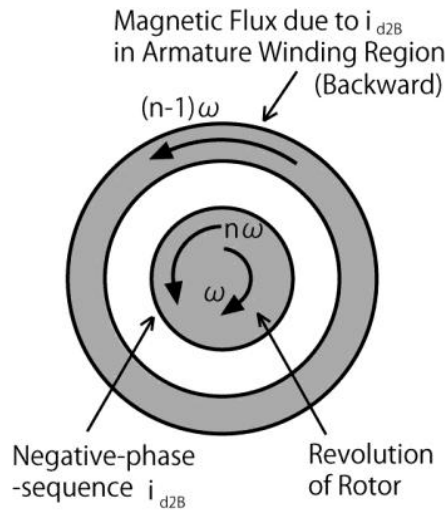

(b) Magnetic flux due to $i_{d 2 B}$

Fig. 3. Magnetic flux in armature winding region

The harmonic voltage $v_{a d}(n-1)$ of the a-phase armature winding is expressed as the following equation.

$$
\begin{aligned}
v_{a d(n-1)}=(n-1) \omega & \cdot M_{a d 2} \cdot\left(I_{d 2 n} / 2\right) \\
& \times \cos \left\{(n-1) \omega t+\phi_{n d}\right\}
\end{aligned}
$$

Consequently, by supplying the AC current with angler frequency $n \omega$ to the winding $\mathrm{d} 2$, the two harmonic voltages with angler frequency $(n+1) \omega$ and $(n-1) \omega$ are generated in the a-phase armature winding.

From (3) and (4), the ratio $|\operatorname{vad}(n+1)| /|\operatorname{vad}(n-1)|$ is as follows.

$$
\left|v_{a d(n+1)}\right| /\left|v_{a d(n-1)}\right|=(n+1) /(n-1)
$$

Therefore, the ratio depends on only $n$, and it becomes constant value.

In order to change separately the amplitudes and the phases of the two harmonic voltages with angler frequency $(n+1) \omega$ and $(n-1) \omega$, the AC voltage $v_{q 2 n}$ with angular frequency $n \omega$ is applied to the winding $\mathrm{q} 2$. Then, the current $i_{q 2 n}$ as shown in (6) flows into the winding q2.

$$
i_{q 2 n}=I_{q 2 n} \cos \left(n \omega t+\phi_{n q}\right)
$$

where $I_{q 2 n}$ is the maximum value of $i_{q 2 n}$ and $\phi_{n q}$ is the initial electrical angle at $t=0$.

As well as the current $i_{d 2 n}$ in the winding $\mathrm{d} 2, i_{q 2 n}$ is resolved into the positive-phase-sequence component $i_{q 2 F}$ and the negative-phase-sequence component $i_{q 2 B}$.

$$
i_{q 2 n}=i_{q 2 F}+i_{q 2 B}
$$

The harmonic voltages generated by $i_{q 2 F}$ and $i_{q 2 B}$ are made to be $v_{a q(n+1)}$ and $v_{a q(n-1)}$, respectively. The angler frequency of $\operatorname{vaq}_{(n+1)}$ is $(n+1) \omega$ and that of $\operatorname{Vaq}_{(n-1)}$ is $(n-1) \omega$.

These harmonic voltages are derived as the following equations, when the spatial phase difference $\pi / 2$ of the winding $\mathrm{q} 2$ for the $\mathrm{d} 2$ winding is considered.

$$
\begin{aligned}
v_{a q(n+1)}= & (n+1) \omega \cdot M_{a q 2} \cdot\left(I_{q 2 n} / 2\right) \\
& \times \cos \left\{(n+1) \omega t+\phi_{n q}+\pi / 2\right\} \\
v_{a q(n-1)}=( & n-1) \omega \cdot M_{a q 2} \cdot\left(I_{q 2 n} / 2\right) \\
& \times \cos \left\{(n-1) \omega t+\phi_{n q}-\pi / 2\right\}
\end{aligned}
$$

where $M_{a q 2}$ is the mutual inductance between the a-phase armature winding and the winding $\mathrm{q} 2$.

By assuming that winding $\mathrm{d} 2$ and winding $\mathrm{q} 2$ are symmetrical two-phase winding, $\operatorname{Mad}_{2}=M_{a q 2}=M_{a 2}$ is set in the following. From (3) and (8), the total voltage $v a(n+1)$ with angler frequency $(n+1) \omega$ in the a-phase armature winding is given as the following equation.

$$
\begin{aligned}
v_{a(n+1)}= & v_{a d(n+1)}+v_{a q(n+1)} \\
= & (n+1) \omega \cdot M_{a 2} \cdot\left[\left(I_{d 2 n} / 2\right) \cos \left\{(n+1) \omega t+\phi_{n d}\right\}\right. \\
& \left.+\left(I_{q 2 n} / 2\right) \cos \left\{(n+1) \omega t+\phi_{n d}+\pi / 2\right\}\right]
\end{aligned}
$$

Moreover, from (4) and (9), the total voltage $v_{a(n-1)}$ with angler frequency $(n-1) \omega$ in the a-phase armature 
winding is given as the following equation.

$$
\begin{aligned}
v_{a(n-1)}= & v_{a d(n-1)}+v_{a q(n-1)} \\
= & (n-1) \omega \cdot M_{a 2} \cdot\left[\left(I_{d 2 n} / 2\right) \cos \left\{(n-1) \omega t+\phi_{n d}\right\}\right. \\
& \left.+\left(I_{q 2 n} / 2\right) \cos \left\{(n-1) \omega t+\phi_{n d}-\pi / 2\right\}\right]
\end{aligned}
$$

By substituting the value of $2 \pi f$ for $\omega$ in (10) and (11), it is possible to use them as the relational expressions of the frequency $f$.

Similarly, the harmonic voltages on the b-phase and the c-phase can be obtained.

\section{Experiment on Harmonic Voltage of Armature Winding}

Fig. 4 shows the circuit diagram for an experiment on the generation of harmonic voltages using the experimental synchronous machine. The frequency $f$ of the power line is $60(\mathrm{~Hz})$. The windings $\mathrm{d} 2$ and $\mathrm{q} 2$ are connected to an oscillator (frequency: $n f$ ). The phases of both currents are able to change separately by the phase shifters shown in Fig. 4. The amplitudes of both currents are also able to be adjusted separately by the power amplifiers.

Fig. 5 shows the power spectrums of the armature winding voltage obtained by an FFT analyzer in the case of supplying currents with the frequency $4 f$ and $6 f$ to only the winding q2 ( $I_{d 2 n}=0 \mathrm{~A}, I_{q 2 n}=0.2 \mathrm{~A}$ ). For the frequency of $4 f$ (Fig. 5(a)), it is confirmed that harmonic voltages of $3 f$ and $5 f$ are generated in the armature winding. Moreover, for the frequency $6 f$ (Fig. 5(b)), harmonic voltages of $5 f$ and $7 f$ are confirmed.

(4) is also applicable for the case of supplying the current to the q-axis winding. By substituting 4 for $n$ in this equation, the ratio of harmonic voltage becomes $5 / 3 \approx 1.67$. Moreover, the ratio is $7 / 5=1.4$ for $n=6$. These values almost agree with the experimental result.

When an AC current with frequency $n f$ is supplied to the winding $\mathrm{d} 2$, induced current flows in the winding $\mathrm{d} 1$ on the same d-axis and the magnetic flux due to the current in the winding $\mathrm{d} 2$ greatly decreases. Therefore, in order to prevent induced current, the reactor shown in Fig. 4 is connected to a circuit for the winding $\mathrm{d} 1$.

However, the reduction of the magnetic flux due to the induced current is occurred, since the inductance value of the reactor is finite.

The maximum value $I_{d 2 n}$ of $\mathrm{AC}$ current $i_{d 2 n}$ in the

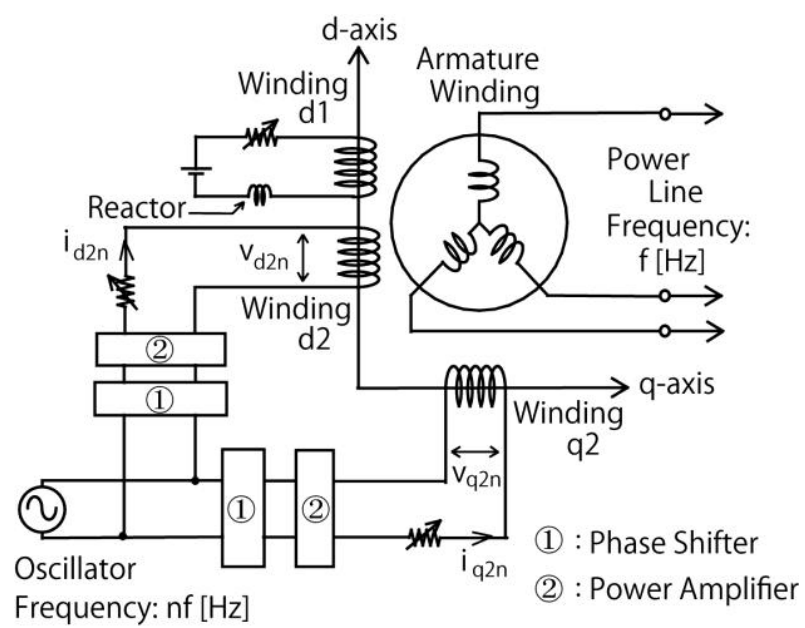

Fig. 4. Circuit Diagram for Experiment

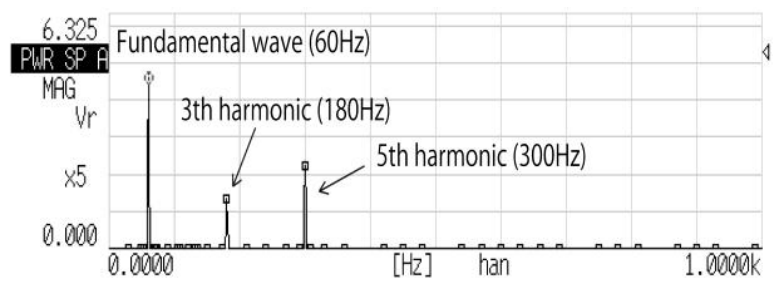

(a) Frequency $4 f$ (current in winding q2)

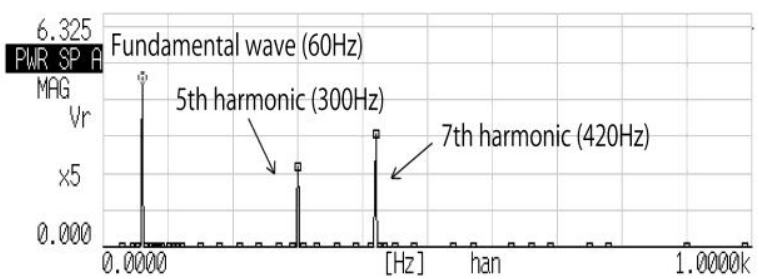

(b) Frequency $6 f$ (current in winding q2)

Fig. 5. Power spectrum of voltage of armature winding $\left(I_{d 2 n} / \sqrt{2}=0 \mathrm{~A}, \quad I_{q 2 n} / \sqrt{2}=0.2 \mathrm{~A}\right)$

winding $\mathrm{d} 2$ can be separated as the following equation.

$$
I_{d 2 n}=I_{d 2 n e}+I_{d 2 n i}
$$

where, Id2ne is effective component, which generates the magnetic flux and $I_{d 2 n i}$ is non-effective component of which the magnetic flux is canceled by the magnetic flux due to the induced current of the winding $\mathrm{d} 1$.

Fig. 6 shows the power spectrums of the armature voltage in the case of the frequency $6 f$ and the current $I_{d 2 n e} / \sqrt{2}=I_{q 2 n} / \sqrt{2}=0.15$ A.

Fig. 6 (a) shows the power spectrum in the case of $\phi_{d n}=\phi_{q n}$. In addition, Figs. 6(b) - 6(d) show the power spectrum in changing the phase of $i_{d 2 n}$ by the phase shifter. These figures indicate that the ratio to the 5 th and 7th harmonic voltages changes with the phase difference 
between $i_{d 2 n}$ and $i_{q 2 n}$

In Fig. 6(c), the 5th harmonic is large compared with the value in other figures, and the 7 th harmonic becomes almost 0 . This is confirmed by substituting $\phi_{d n}=\phi_{q n}+\pi / 2$ for (10) and (11).

In contrast, in Fig. 6(d), the 7th harmonic is large compared with the value in other figures, and the 5th harmonic becomes almost 0 . Similarly, this can also be confirmed by substituting $\phi_{d n}=\phi_{q n}-\pi / 2$ for (10) and (11).

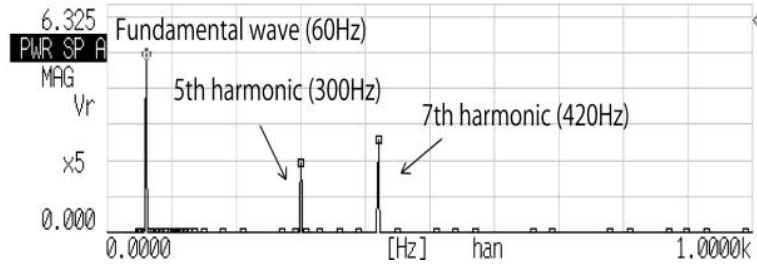

(a) Phase difference: 0

( $i_{d 2 n}$ and $i_{q 2 n}$ are equal to the same phase.)

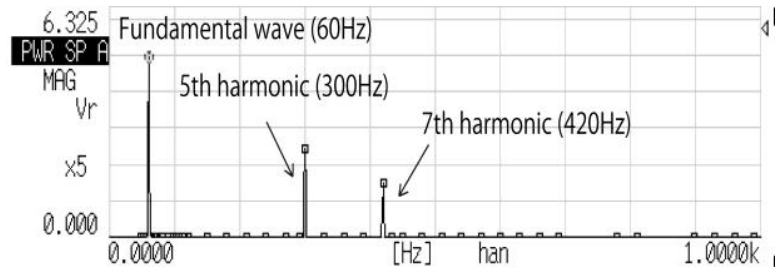

(b) Phase difference: $\pi / 6\left(i_{d 2 n}\right.$ is leading from $i_{q 2 n}$.)

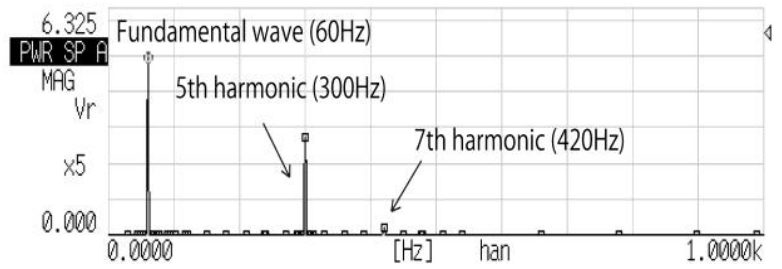

(c) Phase difference: $\pi / 2\left(i_{d 2 n}\right.$ is leading from $i_{q 2 n}$.)

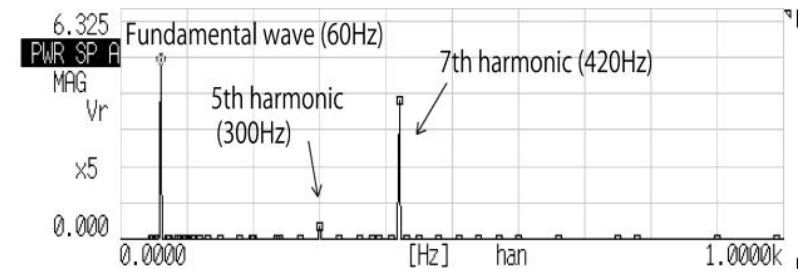

(d) Phase difference: $-\pi / 2$ ( $i_{d 2 n}$ is lagging from $i_{q 2 n}$.)

Fig. 6. Power spectrum of voltage of armature winding $\left(I_{d 2 n e} / \sqrt{2}=I_{q 2 n} / \sqrt{2}=0.15 \mathrm{~A}\right)$

\section{Conclusions}

In this paper, the equations of the harmonic voltage generated in the armature winding are shown in the case of supplying currents with angler frequency $n \omega$ (frequency $n f$ ) to the d-axis and the q-axis harmonic field windings.

We constructed a synchronous machine to demonstrate finally the reduction of the harmonics in a power line. The power spectrum of the armature voltage was measured in the case of supplying the AC currents with frequency $n f$ to the harmonic field windings of the experimental synchronous machine.

The results obtained are as follows:

(1) By supplying AC current with frequency $n f$ to the harmonic field winding, harmonic voltages with frequency $(n-1) f$ and $(n+1) f$ are generated in the armature winding.

(2) The equations of harmonic voltages generated in the armature winding were derived in the case of supplying AC currents with frequency $n f$ to the daxis and q-axis harmonic windings.

(3) The theory and the experiment clarified that the ratios of the amplitudes of the harmonic voltages in the armature winding can be changed in the case of supplying $\mathrm{AC}$ currents to the $\mathrm{d}$-axis and q-axis harmonic windings.

\section{Acknowledgement}

We appreciate to Mr. S. Kawabata and Mr. N. Yoshinaga (students of master course in Sojo-University) for the assistance of the experiment.

\section{References}

[1] R. Uchida, et al., "Power conversion circuits and systems having function of active filter", Technical Report of IEEJ No.643, July 1997

[2] S. Nagano, et al., "Problems and corresponding technics on harmonics of synchronous machine", Technical Report of IEEJ No.903, December 2002

[3] S. Kawabata, E. Mukai, T. Kakinoki, and S. Fukai, "Study on reduction of harmonics using synchronous machine with qaxis field winding", Record of 2010 Joint Conference of Electrical and Electronics Engineers in Kyushu, 07-2P-03, September 2010

[4] S. Kawabata, E. Mukai, T. Kakinoki, H. Yamaguchi, S. Fukai, "On reduction of harmonics in power line using synchronous machine", Record of 2011 Joint Conference of Electrical and Electronics Engineers in Kyushu, 06-2A-02, September 2011 


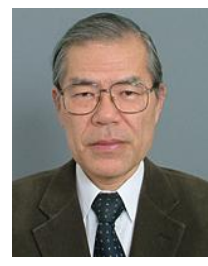

Eiichi Mukai received his B. E. degree from Saga University in 1972. Later, he received his Doctor Eng. degree from Kyoto University. He joined Hitachi Electronics Ltd. in 1972. In 1974 he became a research associate at Saga University, and in 1993, an associate professor. In the same year he became an associate professor at Kumamoto Institute of Technology (Now, Sojo University), and in 1997, a professor. Since then he has been engaged in research related mainly to electrical machinery and apparatus. He is the members of IEEJ, SICEJ and CSSJ.

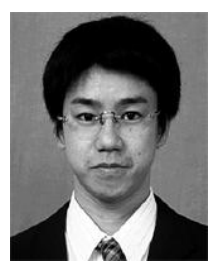

Toshio Kakinoki received his Master's degree from Kagoshima University in 1996 and became an assistant lecturer at Kumamoto Institute of Technology (Now, Sojo University). He became an Associate Professor in 2013 at Sojo University. He is primarily pursuing research on magnetic levitation system. He holds a D. Eng. degree.

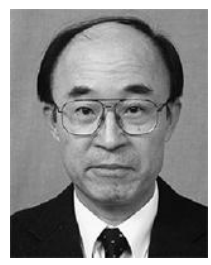

Hitoshi Yamaguchi graduated from the Department of Electrical Engineering at the University of Kyushu in1963 and joined Fiji Denki. He was involved in research on linear motors, rope-less elevators, and magnetic levitation railways. In 1996, he become an instructor at Sojo University, and has been a professor there since 1998. He is primarily pursuing research related to constant conductance-type magnetic levitation railways. He holds a D. Eng. degree.

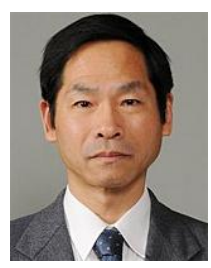

Yoshimasa Kimura received the B.E. and M.E. degrees and the Ph.D. degree from Tokushima University, in 1977, 1979, and 1995, respectively. In 1979, he joined the Nippon Telegraph and Telephone Corporation (NTT), he has been engaged in research and development of Kanji pattern recognition, online handwriting recognition, etc. In 2003, He became a professor at Kochi University of Technology. In 2008, he became a professor at Sojo University. He is the members of the IEEE, IEICE and IEEJ, etc.

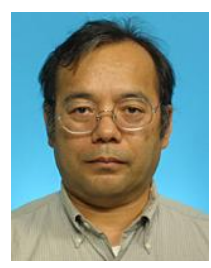

Sumio Fukai graduated in 1978 from Dept of Electronic Engineering, Saga University. Later, he received the Doctor of Engineering from Kyushu University. In 1982 he joined the staff of the Saga University, and in 1995, an associate professor. He has been engaged in research on active RC circuits synthesis and integrated circuit design. He is the members of the IEICE, IEEJ, and IEEE. 